\title{
EDITORIAL
}

\section{Ein breites Themenspektrum}

\author{
Andreas Klee $\cdot$ Hendrikje Wehnert
}

(C) Springer-Verlag Berlin Heidelberg 2012

Das vorliegende Heft der Zeitschrift „Raumforschung und Raumordnung“ beginnt mit einem Beitrag von Horst Zimmermann in der noch relativ neuen Rubrik ,Zur Diskussion“. Mit dieser Rubrik intendieren die Herausgeber der Zeitschrift, akzentuierten Äußerungen und Perspektiven zu aktuellen planungspolitischen und -praktischen Themen, aber auch zu neuen wissenschaftlichen Diskursen einen Ort zu geben. Dadurch sollen auch kontroverse Debatten geführt werden - wie im vorliegenden Heft. Zimmermann bezieht sich in seinem „Zuruf" auf das Schwerpunktheft der Zeitschrift zum Thema „Die gesellschaftliche Konstituierung von Kulturlandschaft“ (Heft 2.2012), das verschiedene Beiträge der Kulturlandschaftsforschung zusammenfasst und dabei eine konstruktivistische Perspektive einnimmt. Zimmermann erweitert diese in der sozial- und raumwissenschaftlichen Forschung derzeit prominente Forschungsrichtung um einen eher nüchternen ökonomischen Aspekt. Er zeigt, dass die Frage, wie sich Kulturlandschaften durch subjektive Bedeutungszuschreibungen im alltagssprachlichen Handeln konstituieren, unvollständig gestellt wurde. Im Schwerpunktheft lag der Fokus auf der soziologischen, politikwissenschaftlichen, planerischen und sozialgeographischen Perspektive. Dieser Diskurs, das zeigt der ,Zuruf“ in diesem Heft, kann sinnvoll erweitert werden, wenn Land-

Dr. A. Klee $(\triangle)$

Akademie für Raumforschung und Landesplanung - LeibnizForum für Raumwissenschaften, Hohenzollernstraße 11, 30161 Hannover, Deutschland

E-Mail: Klee@arl-net.de

H. Wehnert

Leibniz-Institut für ökologische Raumentwicklung, Weberplatz 1, 01217 Dresden, Deutschland

E-Mail: h.wehnert@ioer.de schaft auch als öffentliches, ökonomisches Gut betrachtet wird.

Dennis Guth, Stefan Siedentop und Christian Holz-Rau fragen in ihrem Beitrag „Erzwungenes oder exzessives Pendeln?" nach dem Einfluss der Siedlungsstruktur auf den Berufspendelverkehr. Sie setzen bei der Beobachtung an, dass der Pendelverkehr von Beschäftigten zwischen Wohnund Arbeitsort nach wie vor wächst, fragen dann aber, bis zu welchem Grad dies mit der Siedlungsstruktur erklärt werden kann. Für die Praxis der regionalen und kommunalen Planung ist diese Frage eine entscheidende. Haben sich doch nahezu alle Akteure in der Wissenschaft wie in der kommunalen und regionalen Planung für verkehrssparende Siedlungsstrukturen als ein Planungsziel ausgesprochen. Als Stichworte können hier die Nutzungsmischung oder die „Stadt der kurzen Wege“ genannt werden. Aber wie sieht die Realität tatsächlich aus? Empirisch ermittelte Mobilitätsentwicklung, Pendleraufkommen und zurückgelegte Wege führen zu einem ernüchternden Schluss: Das verkehrssparende Planen und Bauen muss um eine auf individuelle Verhaltensmuster ausgerichtete Politik und Planung ergänzt werden, denn ein guter Teil des Pendelverkehrszuwachses resultiert aus individuellen Standortentscheidungen, die sich eben auch gegen die gut gemeinten planerischen Vorstellungen von verkehrssparender Siedlungsstruktur richten. Offenbar entscheiden sich viele Arbeitnehmerinnen und Arbeitnehmer gegen eine Wohnung am Ort der Arbeitsstätte. Die Gründe dafür sind sicher vielfältig und nachvollziehbar. Die Nähe zur Arbeitsstätte ist da nur ein Faktor unter vielen - Mietpreise, Ausstattungsmerkmale, infrastrukturelle Gegebenheiten und persönliche Bindungen spielen eine ebenso große Rolle. Welche Schlussfolgerungen können daraus gezogen werden? Möglicherweise wissen wir zu wenig über den individuellen Stellenwert der räumlichen Nähe von Wohnung und Arbeitsstätte oder 
- anders ausgedrückt - darüber, ob das Pendeln tatsächlich als „erzwungen“ empfunden wird oder möglicherweise als ein kleines Übel, das gegen andere Rahmenbedingungen individuell ,abgewogen' wird.

Stephan Grohs richtet seinen Blick auf das englische Planungssystem in der Zeit der Labour-Regierungen zwischen 1997 und 2010 und überschreibt seine Analyse mit der Frage „Das Ende des regionalen Experiments?“. Das englische Planungssystem erfuhr in dieser Zeit einen grundlegenden Wandel. Hatten die zuvor regierenden Konservativen die Bedeutung der räumlichen Planung schrittweise zurückgefahren oder ausgehöhlt, erhielt sie wieder mehr Gewicht, beispielsweise durch eine Verknüpfung von Flächennutzungsplanung mit strategischen Planungsansätzen und durch eine Aufwertung der regionalen Ebene. Welche Auswirkungen hatten diese Reformen auf die Steuerungsfähigkeit der Akteure? Und wie wirken sich die Reformen auf die künftige englische Planungspolitik unter zwischenzeitlich wieder veränderten politischen Vorzeichen aus? Grohs analysiert den Implementationsprozess der Reformen und zielt auf eine Bewertung des institutionellen Wandels aus einer steuerungstheoretischen Perspektive.

Das Themenfeld ,demographischer Wandel“ hat mittlerweile in der Zeitschrift ,Raumforschung und Raumordnung“ gute Tradition. Seit vielen Jahren erforschen Autorinnen und Autoren die demographische Situation in Deutschland und Europa, skizzieren die künftigen Entwicklungen, weisen auf die raumbezogenen Implikationen hin und geben Entscheidungsträgern in Politik und Verwaltung Hinweise auf einen konstruktiven Umgang mit den Herausforderungen. Im vorliegenden Heft wird die Perspektive um eine bisher nicht allzu häufig gestellte Frage erweitert: Welche Auswirkungen hat der demographische Wandel auf die Wirtschaftsentwicklung in Schrumpfungsregionen? Anke Matuschewski und Birgit Leick gehen der Frage in der Region Oberfranken aus einer Mikro-Perspektive nach. Sie fördern im Rahmen einer empirischen Erhebung die Einschätzungen der Wirtschaftsakteure zutage, umreißen deren Herausforderungen und mögliche marktbezogene Anpassungsstrategien.

Schließlich beleuchten Andreas Pyschny, Erik Wilde und Robert Knippschild den Kooperationsprozess von Städten und Gemeinden in der Region Halle/Leipzig bei der Entwicklung von Gewerbeflächen. Sie analysieren unter anderem die derzeitigen Arbeits- und Kommunikationsstrukturen und fragen, ob diese Strukturen als eine Form von „Regional Governance" bezeichnet werden können. So viel kann vorweggenommen werden: Die interkommunale Kooperation zur Gewerbeflächenentwicklung weist nur einige Merkmale dessen auf, was in der Fachdiskussion als „Regional Governance" bezeichnet wird. Zahlreiche Elemente fehlen. Dies sollte durchaus als Plädoyer für einen behutsamen Umgang mit dem Governance-Begriff verstanden werden.

Unseren Leserinnen und Lesern wünschen wir eine anregende Lektüre. 